\title{
Robótica educativa: propuesta curricular para Colombia*
}

\section{Gisselle Ximena Pérez Acosta}

https://orcid.org/000o-0002-0965-4903 Universidad Pedagógica y Tecnológica de Colombia

gisselle.perez@uptc.edu.co
Miguel Ángel Mendoza-Moreno

https://orcid.org/0000-0001-9000-5881 Universidad Pedagógica y Tecnológica de Colombia

miguel.mendoza@uptc.edu.co

\section{Resumen}

Este artículo presenta una propuesta de incorporación de robótica educativa para la educación básica y media, como apoyo a las Orientaciones Generales de Educación en Tecnología de Colombia del Ministerio de Educación Nacional, a partir de diferentes aproximaciones a nivel nacional e internacional. La investigación se desarrolló con un enfoque metodológico mixto, con fases documentales, descriptivas, analíticas y proyectivas. La estructura documental integra tres apartados: una revisión conceptual de la robótica educativa; su importancia, a partir de literatura científica y programas aplicados a nivel internacional y nacional; y finalmente, el tratamiento a la robótica desde los ejes de enfoques, perspectivas y dimensiones del saber. Se evidencia la tendencia a incrementar la educación en robótica, tanto en el sector privado como oficial, enfocado al "uso del tiempo libre". En consonancia con esta tendencia, proponemos el diseño de un modelo curricular en espiral que se estructura en cuatro niveles de complejidad, pertinente con los principios de una educación significativa e integradora, acorde con las necesidades y conocimientos de vanguardia para la educación inicial en ciencia y tecnología.

\section{Palabras claves (Fuente: tesauro de la Unesco)}

Colombia; diseño de currículum, innovación educacional; robótica educativa; tecnologías educativas.

* Este artículo es derivado de la tesis de maestría "Esquema gamificado para la adopción de robótica básica en educación primaria”, que será presentada a la Universidad Pedagógica y Tecnológica de Colombia.

Recepción: 15/03/2020 | Envío a pares: 19/08/2020 | Aceptación por pares: 02/09/2020 | Aprobación: 13/10/2020 


\title{
Educational Robotics: Curricular Proposal for Colombia*
}

\begin{abstract}
This paper presents a proposal for incorporating educational robotics into primary and secondary education to support the Ministry of National Education's general guidelines for technology education in Colombia from various national and international approaches. The research is carried out with a mixed-method and divided into the documentary, descriptive, analytical, and projective phases. The paper contains three sections: a conceptual review of educational robotics; its importance, based on scientific literature and applied programs internationally and nationally; and finally, the treatment of robotics from different knowledge approaches, perspectives, and dimensions. We noted a growing tendency to introduce robotics education in private and public schools, focused on the "use of free time." Therefore, we propose designing a spiral curricular model with four complexity levels relevant to the principles of meaningful and inclusive learning, per the current needs for and cutting-edge knowledge of science and technology education.
\end{abstract}

Keyword (Source: Unesco Thesaurus)

Educational robotics; educational technology; curriculum design; educational innovations; Colombia.

* Paper derived from the masters dissertation "Esquema Gamificado para la adopción de Robótica básica en Educación primaria", to be presented to the Universidad Pedagógica y Tecnológica de Colombia. 


\section{Robótica educativa: proposta curricular para a Colômbia*}

\section{Resumo}

Este trabalho apresenta uma proposta de incorporação da robótica educativa na educação básica e média, como apoio para as orientações gerais de educação em tecnologia da Colômbia do Ministério de Educação nacional, a partir de diferentes abordagens no âmbito nacional e internacional. A pesquisa foi desenvolvida com uma abordagem metodológica mista, com fases documentais, descritivas, analiticas e projetivas. A estrutura documental integra quatro seções: revisão conceitual da robótica educativa; sua importância a partir da literatura cientifica; programas aplicados nacional e internacionalmente; por último, tratamento da robótica sob os eixos de abordagens, perspectivas e dimensões do saber. É evidenciada a tendência em aumentar a educação em robótica, tanto no setor privado quanto no público, focada no "uso do tempo livre". Em consonância com essa tendência, propomos um desenho de um modelo curricular em espiral que é estruturado em quatro níveis de complexidade, pertinentes com os princípios de uma educação significativa e integradora, conforme as necessidades e os conhecimentos de vanguarda para a educação inicial em ciência e tecnologia.

\section{Palavras-chave (Fonte: tesauro da Unesco)}

Robótica educacional; tecnologia educacional; desenho de currículo, inovação educacional; Colômbia.

Este artigo é derivado da dissertação de mestrado "Esquema Gamificado para la adopción de Robótica básica en Educación primaria" que será apresentado ao Universidad Pedagógica y Tecnológica de Colombia. 
La sociedad exige la erradicación de las diversas modalidades de analfabetismo y, por ende, el manejo y desarrollo del conocimiento (Pina, 2017). Para ello es vital el fortalecimiento en ciencias, artes, humanidades y tecnología, que demanda para los educandos un cúmulo de habilidades, capacidades y competencias que abarquen una amplia gama de saberes conectados para dar solución a su contexto y a las necesidades globales. Por esto, se insta a las instituciones educativas a reformular sus planes de estudio e incorporar nuevas metodologías y tecnologías de modo que los estudiantes puedan adquirir las destrezas demandadas (Štuikys, 2015, pp. 265-283).

Es así que, complementario a la relevancia que socialmente la tecnología ha asumido, el currículo de tecnología de las escuelas se dinamiza con la implicación de la robótica en su programa, como un método integrador que abarca metodologías novedosas de enseñanza que hacen uso del progreso tecnológico a través de una visión constructivista (Lepuschitz et al., 2018, pp. 100-112). En consecuencia, la robótica educativa se constituye en una herramienta para empoderar a los estudiantes en la era digital y, al vincularse con otras asignaturas y materializar conceptos abstractos, representa una oportunidad en todos los niveles escolares para desarrollar aprendizajes a través de una relación teórico-práctica de saberes (Benitti, 2012; Lepuschitz et al., 2018, pp. 53-64; Scaradozzi et al., 2015).

El proceso investigativo se desarrolló a partir de seis momentos. En primer lugar, una descripción metodológica centrada en la identificación de fuentes esenciales para el estudio; segundo, el abordaje de la relevancia conceptual e integradora de la robótica educativa en el proceso de aprendizaje; tercero y cuarto, la exploración en los contextos internacional y colombiano, respectivamente, del estado actual de la materia, basado en la forma en que se aborda la robótica educativa y los conceptos que integra, clasificando su uso a partir de cinco enfoques; en quinto lugar, la sintesis de los aportes de la propuesta curricular, incluyendo los conceptos, objetivos de aprendizaje y temáticas indispensables para el proceso de enseñanza-aprendizaje de la robótica educativa, basado en un análisis cruzado de enfoques, perspectivas y dimensiones, alineada con otras asignaturas para la educación básica (primaria-secundaria) y media de Colombia; por último, se dan las conclusiones y se perfilan trabajos futuros enfocados en la importancia de la enseñanza de la robótica educativa.

\section{Metodología}

Este trabajo se realizó con un enfoque de investigación mixta, contemplando fases documentales, descriptivas, analíticas y proyectivas (Hurtado, 2000), a partir de la identificación de aspectos y la recolección de datos en diversas fuentes, caracterizando y detallando los aspectos relevantes, para formar criterios de análisis que serán expuestos a nivel general en la propuesta curricular. Estos pasos se complementan con aportes del enfoque cualitativo en la caracterización de la robótica educativa y cómo está permeando a través del currículo los niveles global y local, lo que permite una descripción detallada y depurada del contexto y garantiza objetividad y comprensión (Hernández et al., 2010; Quecedo et al., 2002). También se presenta un enfoque cuantitativo a través de la identificación de aportes gubernamentales y privados brindados por el área de aplicación por medio de la lógica deductiva y recolección de información (Hernández et al., 2010).

Las fuentes que abastecieron el estudio fueron obtenidas de bases de datos científicas, principalmente Scopus, para lo cual se constituyó la cadena de búsqueda "Robótica Educativa" + "Currículo", lo que condujo a obtener un conjunto de documentos que se clasificaron por territorios o naciones, dando como resultado 52 naciones, de los cuales se toman para este estudio 19, que presentan documentos oficiales y artículos relacionados con el currículo en robótica educativa. Cabe aclarar que el resto de los países presentan estudios de casos y ex- 
periencias significativas en robótica educativa, pero no ahondan en las intencionalidades y objetivos del currículo. De los territorios se seleccionó una muestra por cada continente analizando la cantidad de publicaciones que evidencian el nivel de avance.

Posteriormente, siguiendo las fuentes documentales extraídas de las bases de datos científicas, se procedió a identificar las iniciativas cumplidas por diferentes estamentos y el análisis de contenidos digitales, por medio de una búsqueda en entidades como Ministerios o Secretarías de Educación en los países seleccionados, acerca del manejo que le dan a la robótica educativa. Esta información se sistematizó integrando el vínculo de la información, la entidad que regula, los grados que aborda, los objetivos del currículo, los elementos y temáticas que trabaja, así como las observaciones en cuanto a tiempo que vienen desarrollando, los avances presentados y los paradigmas en que se fundamentan. Toda esta información sirvió de base para las diferentes tablas y figuras del artículo.

Adicionalmente, el rigor de la investigación documental se fundamentó en la metodología descriptiva propuesta por Serrano (2000), donde se delimita el grado de avance en lo concerniente a robótica educativa y su currículo, dado que esta temática es amplia y abarca diversas perspectivas: competencias, experimentación, integración de saberes. Con la delimitación anterior, se consolida una ventana de observación de ocho años, en lo concerniente a robótica, y respecto al currículo, se toman trabajos de Colombia de los últimos trece años. Se destaca que este artículo está caracterizado por presentar investigaciones enmarcadas en el área de las tecnologías de la información y las comunicaciones (TIC). De igual forma, se aclara que la recolección de la información se dio a nivel mundial, toda vez que la robótica educativa destaca su auge en países europeos, asiáticos, Estados Unidos $y$, de manera reciente, en países latinoamericanos, en correspondencia con el objetivo investigativo de promover una propuesta curricular que integre las mejores prácticas de la robótica educativa para el modelo colombiano.

Por otro lado, para el diseño de la propuesta curricular se consideró: la forma de tratar didácticamente la robótica a nivel internacional y nacional, el modelo pedagógico constructivista-construccionista, a partir de los aportes brindados por Bruner et al. (1988), en cuanto a su currículo en espiral, y las disposiciones dadas por el Ministerio de Educación Nacional (MEN) de Colombia.

\section{Riqueza conceptual e integradora de la robótica educativa}

Actualmente se ha posicionado el uso de robots en el diario vivir, al punto que este ha sintetizado, evolucionado o revolucionado los procedimientos y aplicaciones a los que se estaba acostumbrado en distintos campos. Conceptualmente, se entiende que los robots son máquinas que integran componentes mecánicos, eléctricos, electrónicos y comunicativos, con un sistema informático para su control a partir de la inteligencia artificial, e internamente aglutinan conceptos físicos, matemáticos, con principios metodológicos de ingeniería a partir del pensamiento algorítmico y el tratamiento de problemas cotidianos (Barrientos et al., 1997; Mestres y Vives, 2011; Saha, 2010).

En consecuencia, desde los años sesenta la robótica se constituyó en un campo de estudio universitario, impartido en talleres y cursos que en los últimos tiempos han brindado aportes en los diferentes niveles de educación, de lo que surgió el concepto de robótica educativa (García et al., 2015; Lepuschitz et al., 2018, pp. 3-15), en respuesta a un interrogante: ¿de qué manera es posible desarrollar procesos formativos que empoderen a los estudiantes para aprender, aplicar y transformar procesos, con base en los aportes de la ciencia y la tecnología, de modo que impacten las situaciones de su diario vivir? Ello derivó en la necesidad de integrar disciplinas como la pedagogía, la psicología, la ingeniería, 
las ciencias, entre otras, para promover la flexibilidad, pertinencia y oportunidad requerida. De esta forma, la robótica educativa se posiciona como una herramienta que posibilita la interdisciplinariedad de forma ilimitada, presentando las ciencias, las matemáticas y la tecnología de una manera lúdica y promoviendo un proceso particular de enseñanzaaprendizaje que potencia y desarrolla tanto las habilidades como las competencias de los educandos. Esto es posible porque la robótica educativa facilita la aplicación de principios científicos, potencia las habilidades de investigación y la resolución de problemas, a partir de estrategias centradas en el razonamiento lógico, analítico y el pensamiento crítico, por medio de la creatividad (Alimisis et al., 2017; Lepuschitz et al., 2018; Merdan et al., 2017; Viegas y Villalba, 2017).

De acuerdo con Mubin et al. (2013), la robótica tiene gran potencial para mejorar el proceso de enseñanza-aprendizaje en el aula, lo que ha generado una serie de investigaciones con diversos enfoques, evaluaciones, usos y aportaciones contextuales, dado que su teoría parte del constructivismo y establece que el aprendizaje de los estudiantes inicia a partir de lo que ellos saben y experimentan. Además Papert (1980) introduce la noción del construccionismo y aclara que el aprendizaje ocurre cuando el educando construye un artefacto tangible y reflexiona sobre su experiencia en la resolución de problemas, de modo que resulta personalmente significativo, por el constante cuestionamiento de los hechos que lo motivaron a construir dicho objeto, con lo cual se genera un aprendizaje cíclico y acumulativo.

De acuerdo con esta concepción, se verifica que la mayoría de los planes de estudio de robótica se enfocan en la práctica y la resolución de problemas, animando a los estudiantes a "aprender haciendo", y se caracterizan por un compromiso activo que les permite desarrollar una comprensión conceptual y creativa a partir del constante diseño y ejecución de prototipos y/o construcciones, media- dos por equipamientos específicos y fraccionando las tareas complejas en más pequeñas para generar andamiaje (Alimisis et al., 2017; Lepuschitz et al., 2018; Merdan et al., 2017; 2020).

De igual forma, hay que considerar a los estudiantes como "consumidores tecnológicos", por lo que aprender con robótica les proporciona oportunidades para cuestionarse, pensar, descubrir, experimentar, diseñar, construir, programar y documentar. Esto forma sujetos reflexivos y autodidactas que relacionan todo su conocimiento con un objetivo concreto, por lo cual no solo aprenden cómo funciona la tecnología, pues también aplican sus habilidades de una manera significativa a partir del trabajo en grupo y las relaciones de andamiaje, donde se transmiten, reciben, asocian y construyen los conocimientos (Khine, 2017; Torres, 2015). Así, la robótica se convierte en un detonante de invaluables efectos en los procesos formativos y en una oportunidad didáctica sin igual para dinamizar los enfoques pedagógicos.

\section{Avances de la robótica educativa: revisión global}

La robótica educativa se ha posicionado como una tendencia, por cuanto el interés científico centra sus esfuerzos no solo en estructurarla en los entornos académicos, sino en explotar su riqueza como recurso integrador. Es por ello que los países, a través de sus Ministerios o Secretarías de Educación, empiezan a implementarla de forma interdisciplinar, al tiempo que empresas privadas se unen al proceso para presentar elementos motivadores y la búsqueda de conocimientos básicos para asumir posturas y actitudes tecnológicas que los encaminen al cambio (Viegas y Villalba, 2017). En este contexto, este trabajo se enfoca en el currículo y planes de estudio de la robótica educativa a nivel mundial, a partir de la inspección de iniciativas en diversos países que den cuenta de acercamientos a la enseñanza de la robótica, ya sea a nivel interdisciplinario o como asignatura individualizada. Con tales propósitos destacan: 
Robotics in Education, serie que presenta investigaciones de esta temática a partir de la Conferencia Internacional sobre Robótica en Educación (RiE) que se celebra anualmente, tomando como referencia las dos últimas ediciones: en Viena (Austria, 2016) y en Sofía (Bulgaria, 2017) (Lepuschitz et al., 2018). Estas publicaciones contemplan metodologías de enseñanza de la robótica, así como diversos planes educativos y su evaluación, midiendo su impacto. Además presentan diseños y análisis de entornos de aprendizaje, enfoques de programación, nuevas aplicaciones, últimas herramientas, sistemas y componentes para el uso de la robótica que se aplican desde la escuela elemental hasta el nivel posgradual, todo ello con un enfoque técnico y didáctico.

Educational Robotics in the Makers Era: Conferencia de Edurobotics, que se integra a Teaching Robotics and Teaching With Robotics (TRTWR), evento que se celebra cada dos años. Se registra el penúltimo tomo, denominado Atenas 2016 (Alimisis et al., 2017), que aborda el papel de la robótica educativa en lo concerniente a la parte tecnológica y su relación con las teorías-metodologías de aprendizaje sugeridas por el constructivismo-construccionismo (Piaget y Papert), además de su relación con Ciencia, Tecnología, Ingeniería, Artes y Matemáticas o STEAM (Science, Technology, Engineering, Art y Mathematics), para generar interés tecnológico, pensamiento creativo, trabajo en equipo y habilidades para resolver problemas tanto en la academia como en la industria.

Por su parte, Robotics in STEM Education. Redesigning the Learning Experience (Khine, 2017) integra una serie de artículos relacionados con enfoques recientes en el avance de la educación en ciencia, tecnología, ingeniería y matemáticas con el uso de robótica, usando métodos innovadores para integrarla en las asignaturas escolares a través de actividades extraescolares y en el aula, además de darle relevancia como herramienta educativa para el proceso de aprendizaje. Esto conlleva, por supuesto, una renovación de los planes de estudio a través de estrate- gias efectivas y tendencias emergentes en su uso, junto con el diseño de actividades de aprendizaje y la afectación de intereses y logros en los estudiantes.

Adicionalmente, durante el segundo semestre de 2018 en este estudio se consultó información de dieciocho países que presentan un adelanto con respecto a la forma en que abordan la robótica educativa, a través de una revisión de diversos enlaces (40) y clasificando la información encontrada en aspectos tales como: Links de revisión, entidad que aborda esta temática, grados escolares en los que se implementa, objetivos de su implementación, elementos y algunos contenidos curriculares que aborda, y observaciones. Se destaca que los enlaces consultados fueron gubernamentales y también se accedió vía online cuando la información remitía a entidades privadas.

Ahora bien, para el avance del estudio a partir de las revisiones de productos científicos y normativos en diferentes países, se caracteriza la robótica educativa en cinco enfoques, que surgen al identificar similitudes en los diferentes comportamientos, intencionalidades, estándares y modalidades de trabajo de los países, además de la revisión bibliográfica. Se evidencia así un tratamiento diverso de la robótica en las escuelas, en cuanto a sus manifestaciones curriculares, toda vez que, cuando se incluye la robótica en dinámicas escolares, se debe realizar un diseño curricular definido a través de un plan de estudios que otorgue importancia al diseño de material de aprendizaje (Bravo y Forero, 2012; Monsalves, 2011; Mubin et al., 2013). De esta manera, se definen y describen los enfoques a continuación:

1. Descriptivo funcional: hace referencia a la libertad que tiene cada nación e institución educativa de dar tratamiento a los conceptos, siempre y cuando los estudiantes se empoderen de la robótica a partir de su comprensión, descripción, función y aplicación, desarrollando conceptos tecnológicos y habilidades técnicas desde temprana edad; por eso se trabaja como materia es- 
colar o disciplina tecnológica (Lepuschitz et al., 2018, pp.100-112; Merdan et al., 2017, pp.169-180).

2. De competencias dicitales: enfoque centrado en el desarrollo de competencias necesarias para que los estudiantes apropien y usen los nuevos lenguajes producidos por las tecnologías de la información y la comunicación, por lo que se busca entender y aplicar los principios y conceptos fundamentales de las computación, ingeniería y diseño, de forma eficiente y comprensible, utilizando la robótica como un medio, dado que estas se consideran competencias del siglo XXI (Alimisis et al., 2017; Heiner, 2018; Scaradozzi et al., 2015; Štuikys, 2015, cap. 12).

3. SOCIAL: consiste en utilizar la tecnología robótica para el bienestar de la ciudadanía, dando realimentación constante, debido a que se generan conocimientos y experiencias a partir de situaciones problema y su respectiva solución y se tienen en cuenta las necesidades que observan los estudiantes en su contexto; adicionalmente, el enfoque los pone en un rol responsable, competente y creativo en el manejo de las TIC, con lo que desarrollan capacidades sociales (Alimisis et al., 2017, pp. 158-169; Heiner, 2018; Merdan et al., 2017, pp. 29-41).

4. InTEGRADOR De SABeres: se toma la robótica como recurso educativo transversal para estimular y mejorar el conocimiento de forma interdisciplinar, ya que presenta una relación simbiótica de forma natural con otras áreas, en especial matemáticas, ciencias, tecnología e ingeniería (STEM), y adicionalmente desarrolla habilidades, dependiendo del énfasis que se le quiera otorgar, pero basado en una educación integral (Merdan et al., 2017, pp. 3-14, 41-53).

5. De uso del tiempo libre: contempla el desarrollo de la robótica atendiendo a un centro de interés o como extraclase, mediado por la interdisciplinariedad, donde los estudiantes asisten a estos espacios extracurriculares de manera vo- luntaria, buscando una profundización según sus intereses (Alimisis et al., 2017, pp. 246-250; Heiner, 2018).

Es preciso mencionar que estos enfoques se pueden relacionar entre sí y que, por tal motivo, puede observarse más de un enfoque, tal como se representa en la Tabla 1, que muestra una clasificación de los países según los enfoques, lo que permite evidenciar una mayor frecuencia en la orientación de la robótica hacia: las competencias digitales, la integración de saberes y, en tercera instancia, lo social.

Tabla 1. Enfoques de la robótica educativa en diversos paises

\begin{tabular}{|c|c|c|c|c|c|}
\hline Pais & 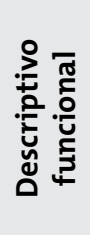 & 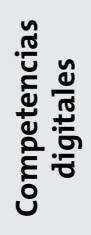 & 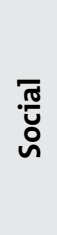 & 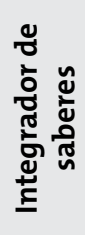 & 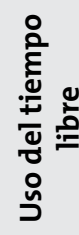 \\
\hline España & $x$ & & $x$ & & \\
\hline Estonia & & $x$ & & $x$ & \\
\hline Inglaterra y Gales & & $x$ & & & \\
\hline Alemania & & & $x$ & $x$ & \\
\hline Finlandia & & & & $x$ & \\
\hline Portugal & $x$ & $x$ & & $x$ & \\
\hline Francia & & $x$ & & & \\
\hline Rusia & & $x$ & & $x$ & \\
\hline Japón & & & $x$ & $x$ & \\
\hline Corea del Sur & & & $x$ & $x$ & \\
\hline China & $x$ & $x$ & $x$ & & $x$ \\
\hline Estados Unidos & & & & $x$ & $x$ \\
\hline México & & $x$ & $x$ & & \\
\hline Salvador & & & & $x$ & \\
\hline Panamá & $x$ & $x$ & & & \\
\hline Brasil & $x$ & $x$ & $x$ & & $x$ \\
\hline Perú & & & $x$ & & \\
\hline Argentina & & $x$ & & & \\
\hline Total & 5 & 10 & 7 & 9 & 3 \\
\hline
\end{tabular}

Fuente: elaboración propia. 
Cabe señalar que la mayoría de las naciones dan tratamiento a varios enfoques, ya que su uso integrado otorga relevancia a la robótica en el campo educativo y permea todos los grados de escolaridad, bien sea como herramienta, como complemento del área de tecnología, o independientemente. Además, se observa que es una apuesta que hacen los gobiernos, entidades privadas y universidades para la formación de profesores y jóvenes, puesto que tras sus investigaciones se detecta un aumento y mejora del proceso de aprendizaje contextualizado y de las capacidades individuales, que se integran a diversas áreas del conocimiento con habilidades y relaciones eficientes con la tecnología y la sociedad (Alimisis et al., 2017, pp. 179-188; Heiner, 2018; Khine, 2017, caps. 1-2; Štuikys, 2015). Así pues, la inspección presentada afianza el hecho de que es deseable para el mundo asumir un enfoque multidimensional de la robótica en su implicación con el proceso formativo.

De igual forma, también se determina que las actividades en robótica usualmente se desarrollan haciendo uso de kits brindados generalmente por el gobierno o por convenios, y desde la informalidad académica en campamentos y actividades extraclase (Karp y Maloney, 2013; Montironi et al., 2015; Ucgul y Cagiltay, 2014). Esto devela la ausencia de una estructura académica que pueda ser replicable, escalable y extensible en favor de la comunidad educativa y los macro currículos.

Adicional al análisis centrado en los enfoques, se presta atención a algunas temáticas que trabajan diversos países (Tabla 2). Es de notar que la mayoría de las naciones registradas en el estudio dan un alto grado de importancia a todo lo concerniente a lógica de programación y uso de lenguajes computacionales (89\%), seguido, con un $67 \%$, de: conceptos integradores, que hacen alusión al uso de otras asignaturas y su respectiva relación entre sí; robótica y sociedad (en lo social, histórico, ético y lo relativo a la ciudadanía y la legislación); y automatización, que integra sistemas de control para el manejo y desarrollo de elementos autónomos. Cabe destacar que la automatización presenta substancialmente conceptos mecánicos, electrónicos, uso de energías, máquinas, diseño, construcción, entre otros, los cuales también se encuentran en la Tabla 2, pero con menor porcentaje; así mismo, la mitad de los países integrados en el estudio tratan de establecer una relación directa entre la robótica y la resolución de problemas como método de aprendizaje.

Tabla 2. Porcentaje de temáticas según países consultados

\begin{tabular}{|c|c|}
\hline Temáticas & Porcentaje (\%) \\
\hline Programación & 89 \\
\hline Mecánica & 56 \\
\hline Electrónica & 39 \\
\hline Conceptos integradores & 67 \\
\hline Resolución de problemas & 50 \\
\hline Máquinas & 56 \\
\hline Robótica y sociedad & 67 \\
\hline Diseño & 50 \\
\hline Construcción & 56 \\
\hline Automatización & 67 \\
\hline Energía & 50 \\
\hline Estructuras & 56 \\
\hline
\end{tabular}

Fuente: elaboración propia.

La relación de la información sintetizada en las Tablas 1 y 2 permite abstraer la información dispuesta en la Figura 1, en la que se observa el impacto de cada temática en los enfoques y el nivel de profundidad con el cual se están ocupando. Se destaca que el enfoque descriptivo funcional no aborda formalmente los conceptos relacionados con energía o estructuras y que cada tema varía según el enfoque, y aunque los países no están ligados a un único enfoque, estos si varían con respecto a la temática, por lo cual su proceso de homogenización resulta complejo. 


\section{Figura 1. Relación entre enfoques y temáticas por número de países que los trabajan}

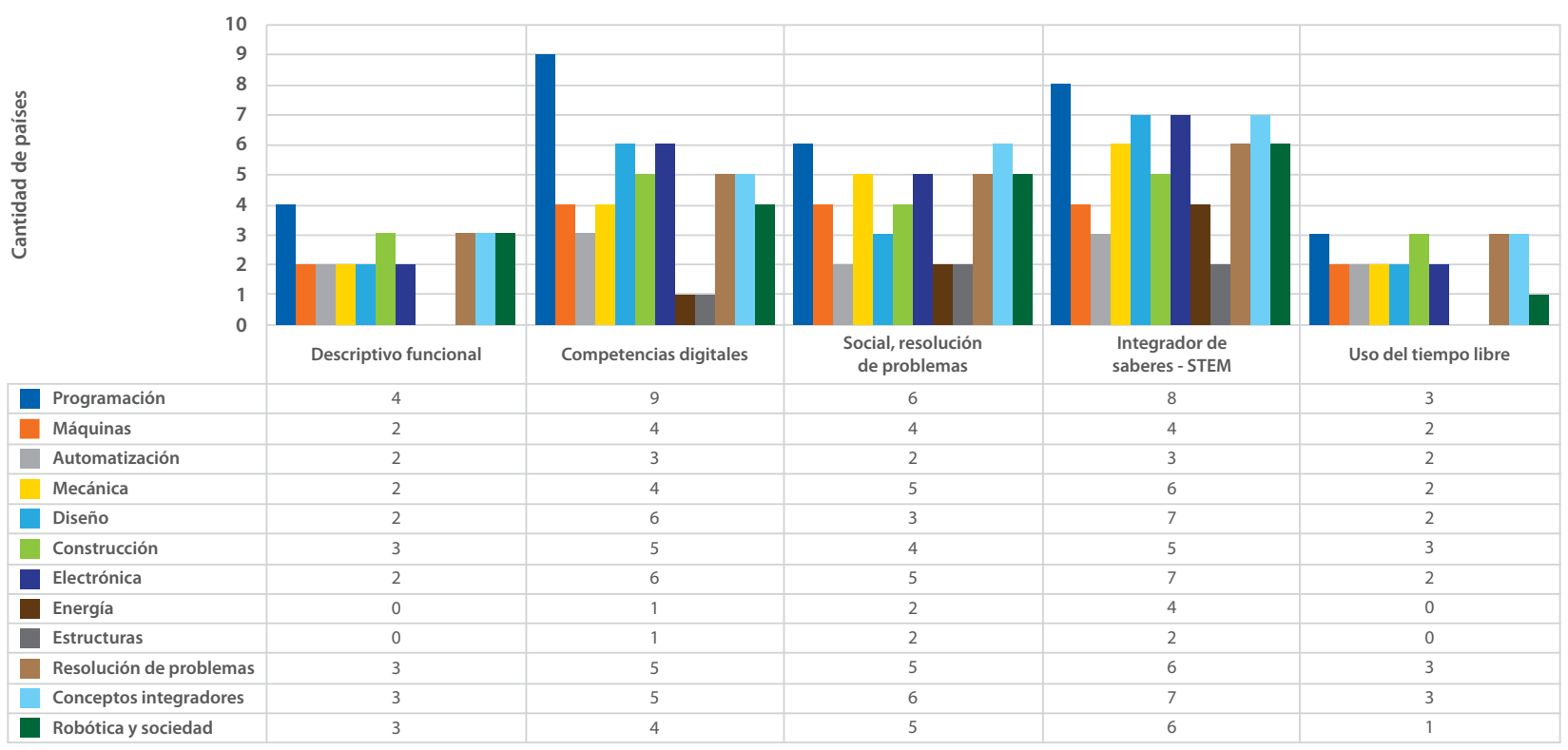

Fuente: elaboración propia.

\section{Actualidad de la robótica educativa para el contexto colombiano}

En lo referente al tratamiento de la robótica educativa en Colombia, se presentan investigaciones a partir de trabajos de grado de licenciaturas, ingenierías, maestrías o de grupos universitarios -como los realizados por Castiblanco et al. (2015), Correa et al. (2019), García et al. (2015), Gómez et al. (2019), Gutiérrez (2016), Meza (2018) y Pinto et al. (2010)-, en los que se provee una descripción de cómo las instituciones están empezando a trabajar la robótica educativa como eje integrador y lúdico del proceso de aprendizaje; esto se da a partir de estudios de caso, que principalmente abordan la robótica como elemento complementario a la jornada escolar o integrado al desarrollo de competencias interdisciplinares, vinculando nuevos agentes, como empresas privadas que desarrollan estrategias semiestructuradas por su condición de informalidad (lo que puede considerarse como el conjunto de las "buenas prácticas").
Colombia cuenta con una amplia gama de instituciones, empresas privadas o públicas que ofrecen el servicio de robótica educativa, de las cuales fueron contactadas 35 durante 2018, a través de sus páginas web o redes sociales, caracterizándolas por: nombre y link; ciudad donde laboran; clasificación de la empresa (pública, privada o convenio); misión, visión y objetivo; tipo de servicio educativo que suplen (formal o no formal); temas y formas en la que los aborda; experiencia; y características organizativas. Con la anterior información se obtuvo como resultado lo observado en la Figura 2, que hace alusión a la clasificación de la empresa, mientras que la Figura 3 muestra el tipo de servicio educativo que suplen tales empresas. Cabe destacar que estas, y en algunos casos instituciones educativas, se encuentran principalmente en las ciudades de mayor población y acceso a las tecnologías y trabajan en ámbitos extraescolares.

Adicionalmente, se observa que las empresas o entidades educativas contactadas bajo revisión de 
sus perfiles online, trabajan en su mayoría el marco STEM, hacen uso de kits robóticos (Lego, Fischer-Technik, Sphero, Arduino, entre otros), manejan lenguajes de programación principalmente gráficos (Scratch, S4A, Alice, Minecraft, entre otros), tienen la modalidad de cursos vacacionales o extracurriculares; sin embargo no se encuentran estandarizadas y no dan certificación, razón por la que estos temas se ven sujetos a la autonomía de las entidades, sin tener unas competencias y temáticas curriculares homogenizadas, y como no se define una estructuración curricular explícita, se puede inferir que en Colombia se trabaja mayormente el enfoque de aprovechamiento del tiempo libre, seguido por el de competencias digitales y el integrador de saberes, pero no desde la institucionalidad ni reglamentado oficialmente por el Ministerio de Educación Nacional.

Figura 2. Tipo de empresas que involucran robótica educativa

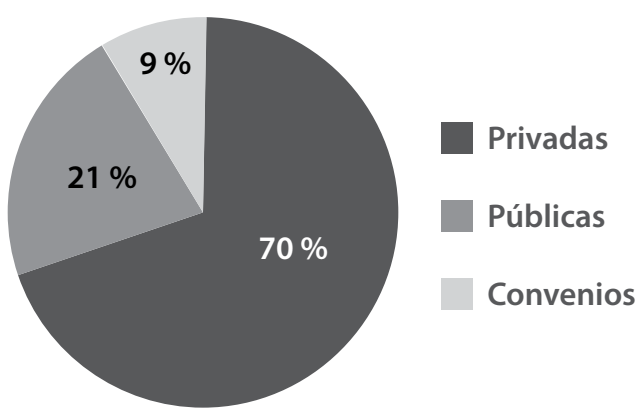

Fuente: elaboración propia.

Figura 3. Servicios educativos que se ofrecen

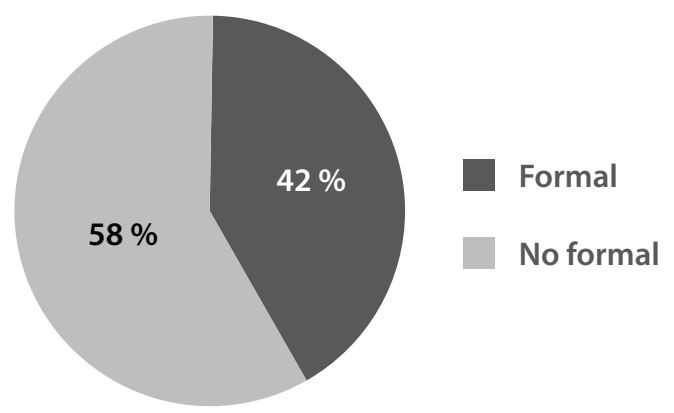

Fuente: elaboración propia.

\section{Propuesta de estándares curriculares de robótica para la educación básica y media en Colombia}

La globalización y los avances tecnológicos afectan a todos los agentes intervinientes en el proceso educativo, mientras que surgen estándares curriculares que evalúan y mejoran los saberes y capacidades comunes de los educandos a través de su escolaridad, pero respetando la autonomía institucional, lo cual da cuenta de una transversalidad de conocimientos. En consecuencia surgen: diseños curriculares, planes de estudio y otras actividades inherentes al quehacer pedagógico (MEN, 2006; 2008).

Como resultado, se puede incluir la robótica en las dinámicas escolares por medio del estándar curricular de tecnología e informática (TI) que responde a una reflexión crítica de la relación entre tecnología y sociedad por medio de la compresión, participación y deliberación para la alfabetización tecnológica (Fundación Compartir, 2015; MEN, 2013), a la que se le otorga importancia, por medio de varios agentes (docentes, educandos, universidades, proveedores de kits, gobierno, entre otros), de forma activa y a través de relaciones multidireccionales y mediadas por elementos motivadores y cognitivos, que involucran asumir posturas y actitudes tecnológicas (Viegas y Villalba, 2017).

Adicionalmente, algunas investigaciones consideran que la implementación de la enseñanza de la robótica debe darse a temprana edad, debido a que los estudiantes de estos grados se encuentran en una etapa donde presentan fuerte entusiasmo y están naturalmente dispuestos y equipados con las debidas capacidades-habilidades cognitivas y de trabajo individual y grupal para afrontar temáticas inherentes a la robótica (Hrbacek et al., 2013; Kandlhofer et al., 2014; Viegas y Villalba, 2017).

Por consiguiente, después de hacer una revisión y análisis para los contextos internacional y nacional del estado de implementación de la robótica -en los que se establecieron los enfoques y concep- 
tos que se manejan mayoritariamente y se revisaron varios casos de estudio que se concentran en diseñar un plan de estudio para una población específica-, y comprendiendo la importancia de la tecnología en la educación colombiana, se propone que su enseñanza debe partir de la integración consciente y estructurada de los enfoques: social, competencias digitales e integrador de saberes, a partir de tres perspectivas de conocimiento: 1) inherentes al área, 2) interdisciplinares y 3) sociales o centrados en habilidades blandas, como se muestra en la Tabla 3, donde se presenta una clasificación de las temáticas a partir de las tres perspectivas mencionadas.

Tabla 3. Relación entre perspectivas del conocimiento y temáticas

\begin{tabular}{|c|c|}
\hline $\begin{array}{l}\text { Perspectiva de } \\
\text { conocimiento }\end{array}$ & Temáticas \\
\hline Inherentes al área & $\begin{array}{c}\text { Mecanismos } \\
\text { Energía } \\
\text { Electrónica / Sensores- } \\
\text { Actuadores }\end{array}$ \\
\hline interdisciplinares & $\begin{array}{c}\text { Programación } \\
\text { Estructuras } \\
\text { Construcciones-Materiales } \\
\text { Conceptos integrados }\end{array}$ \\
\hline $\begin{array}{c}\text { Sociales o centrada en } \\
\text { habilidades blandas }\end{array}$ & $\begin{array}{l}\text { Trabajo en grupo } \\
\text { Solución de problemas } \\
\text { Robótica y sociedad }\end{array}$ \\
\hline
\end{tabular}

Fuente: elaboración propia.

De igual forma, las perspectivas y enfoques que se le otorgan a la robótica educativa deben acompañarse de las siguientes dimensiones: del saber, que hace alusión a la parte cognitiva o los conocimientos que el estudiante adquiere en su formación; del hacer, encargada de la parte práctica o de aplicación, una vez se alcance lo cognitivo; y del ser, atenta al desarrollo humano a partir de las emociones que se adquiere durante la formación.

El modelo curricular en espiral aquí propuesto, basado en el ideado por Bruner (1988), se plantea en tres ejes: ancho, largo y en profundidad, donde el primero se refiere a los enfoques (social, compe- tencias digitales e integrador de saberes), que surge de la necesidad de afrontar la robótica; el segundo hace alusión a las perspectivas del conocimiento (inherentes al área, interdisciplinar y social), que son las temáticas que puede abordar; y el último, que corresponde a las dimensiones del saber, hacer y ser, que son las competencias de aprendizaje para la solución de situaciones a partir de las habilidades y comportamientos. Esto se evidencia al estar relacionados y ligados todos los ejes entre sí, ya que giran en consonancia, en pro del aprendizaje centrado en el estudiante, como se evidencia en la Figura 4.

\section{Figura 4. Ejes del modelo curricular en robótica educativa}

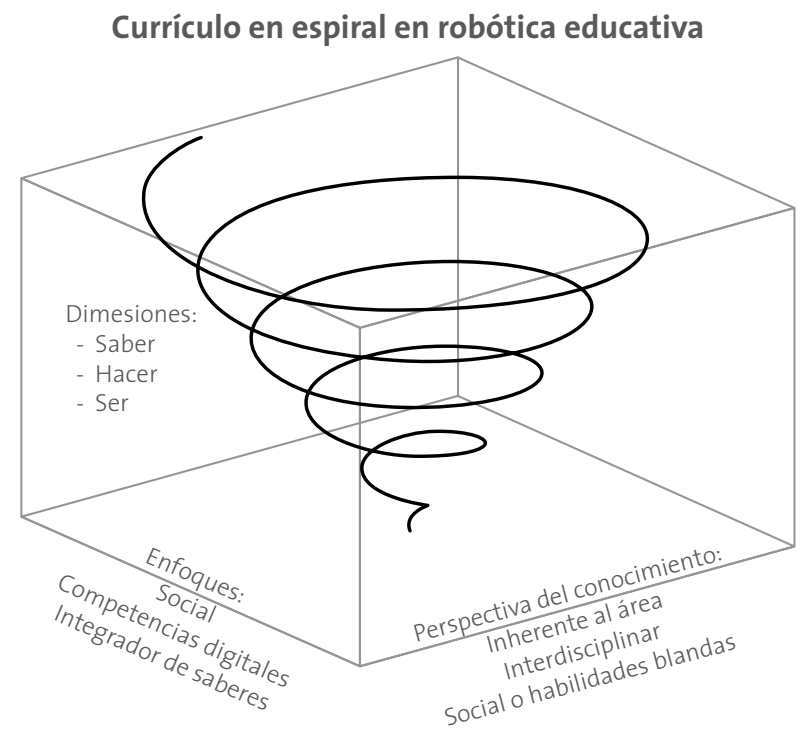

Fuente: elaboración propia.

Conviene destacar que la Tabla 3 se corresponde con la Figura 4 y presenta temáticas generales que se pueden aplicar a diversos grados de escolaridad, ya que se busca: tener continuidad entre las temáticas, aumentar su grado de complejidad al subir el nivel escolar, promover un constante andamiaje y construir saberes, ya que esta propuesta busca la integración de los enfoques. Este currículo presenta como beneficio que ayuda a motivar al estudiante, dado que se trabaja contando con su conocimiento, 
capacidades y recursos, y generando además una conciencia social donde se aumenta su conocimiento general y se lo prepara para la vida profesional (Cantú y Farines, 2007).

El currículo en espiral, especialmente en tecnología robótica, adquiere relevancia, dado que los contenidos tecnológicos cambian continuamente (Cantú y Farines, 2007), los conocimientos van de lo simple a lo complejo y la organización de los mismos se da a partir de una sistematización de temáticas que van relacionadas entre sí. Además, esta comprensión de temáticas es requisito fundamental para tratar nuevos problemas o conocimientos que se encontrará el estudiante dentro o fuera del curso, a partir de sus posibilidades y capacidades individuales y grupales, dado que el aprendizaje en niveles superiores se basa en anteriores, a partir de un constante refuerzo y significatividad, retomando y dándole profundidad a los mismos. Por lo anterior, se proponen cuatro niveles de desarrollo, con sus objetivos generales de aprendizaje, como se muestran en la Figura 5, considerando que la diferencia entre los niveles 1 al 4 radica en que, en consonancia con el modelo en espiral, es el estudiante quien integra cada vez mayores capacidades y elementos tecnológicos para generar soluciones a problemas más complejos, pero, sobre todo, en contextos más diversos.

En el esquema de la Figura 5, apoyada con la Tabla 3, se promueve no una sumatoria de contenidos sino una combinatoria, que ayude al estudiante a hacer uso de sus habilidades para valerse de conocimientos y destrezas de diversas disciplinas a nivel teórico y práctico, considerando diferentes opciones al momento de resolver problemas, con la capacidad de transferir dichas habilidades de una situación a otra, argumentando y dando cuenta de ello. Con este fin, los objetivos propuestos se basaron en la taxonomía de Bloom en la era digital ( $\mathrm{Da}$ Costa et al., 2014; Pinto et al., 2016; Villalonga et al.,

\section{Figura 5. Niveles de robótica educativa en la escuela con sus objetivos generales}

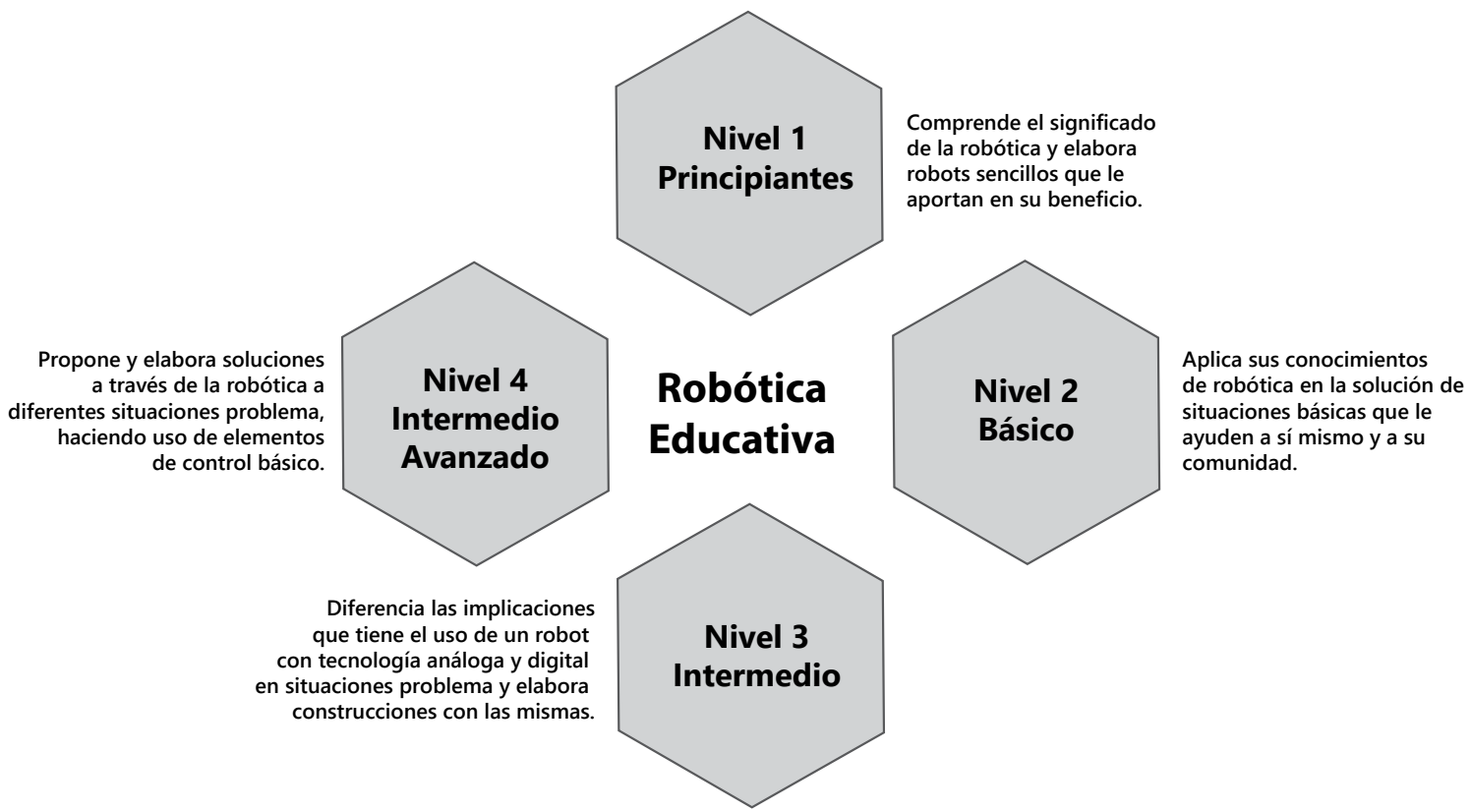

Fuente: elaboración propia. 
2015), donde se tienen en cuenta las dinámicas del siglo XXI y su relación con el aprendizaje. Por ello, el estudiante debe: recordar, comprender, aplicar, analizar, evaluar y crear a partir de entornos mediados por las tecnologías, sin olvidar los aspectos psicomotrices y afectivos que interfieren en el proceso de aprendizaje del estudiante.

Considerando la Figura 5 y la Tabla 3, se presenta un ejemplo de secuenciación de las temáticas (Tabla 4), donde se observan conceptos subyacentes a cada una, para que, al pasar cada nivel, el estudiante aumente y mejore su conocimiento, a partir de subtemas, que pueden ser medibles y evaluables según la profundidad y necesidad del docente, ya que este determina la orientación, a partir de la relación de las temáticas con la robótica. Se destaca que la perspectiva social (y habilidades blandas), en cuanto a la solución de problemas y trabajo en grupo, se corresponde con la metodología y la estrategia para afrontar cada nivel, al igual que los conceptos integrados, que pertenecen a la perspectiva interdisciplinar, ya que van inmersos en todo el proceso.

Cabe destacar que esta propuesta apoya las orientaciones generales de educación en tecnología Guía 30 (MEN, 2008) y le da un peso significativo al papel de la robótica, no solo en la asignatura

Tabla 4. Ejemplo de secuenciación de temáticas por nivel

\begin{tabular}{|c|c|c|c|c|}
\hline Temática & Nivel 1 & Nivel 2 & Nivel 3 & Nivel 4 \\
\hline Mecanismos & $\begin{array}{c}\text { Herramientas } \\
\text { Piezas } \\
\text { Identificación de una } \\
\text { máquina }\end{array}$ & Máquina simple & Máquina compuesta & $\begin{array}{l}\text { Máquinas } \\
\text { electromecánicas }\end{array}$ \\
\hline Energía & $\begin{array}{c}\text { Fuentes de energía y } \\
\text { recursos }\end{array}$ & $\begin{array}{c}\text { Transformación de la } \\
\text { energía }\end{array}$ & $\begin{array}{c}\text { Conservación de la } \\
\text { energía }\end{array}$ & $\begin{array}{c}\text { Manifestación de la } \\
\text { energía a diversas } \\
\text { escalas }\end{array}$ \\
\hline Electrónica & Circuitos básicos & Electrónica análoga & Electrónica digital & $\begin{array}{l}\text { Microcontroladores } \\
\text { Sistemas embebidos }\end{array}$ \\
\hline $\begin{array}{l}\text { Sensores- } \\
\text { Actuadores }\end{array}$ & $\begin{array}{l}\text { Concepto básico de } \\
\text { sensor y actuador } \\
\text { Relación: el cuerpo } \\
\text { humano tiene } \\
\text { sensores y actuadores } \\
\text { Sistemas on-off }\end{array}$ & $\begin{array}{c}\text { Identificar situaciones } \\
\text { que requieran el } \\
\text { uso de sensores y } \\
\text { actuadores y hacer } \\
\text { uso de ellos } \\
\text { Sistemas de tres } \\
\text { posiciones }\end{array}$ & $\begin{array}{l}\text { Conversión de señales } \\
\text { entre el mundo físico } \\
\text { y el entorno del robot }\end{array}$ & $\begin{array}{c}\text { Lectura, manejo e } \\
\text { interpretación de } \\
\text { sensores y actuadores }\end{array}$ \\
\hline Programación & $\begin{array}{l}\text { Secuencias } \\
\text { jerarquías }\end{array}$ & Condicionales y ciclos & $\begin{array}{c}\text { Algoritmos } \\
\text { Lógica booleana }\end{array}$ & $\begin{array}{c}\text { Lógica de } \\
\text { programación } \\
\text { estructurada para } \\
\text { cualquier lenguaje }\end{array}$ \\
\hline Estructuras & $\begin{array}{l}\text { Desarrollo de } \\
\text { motricidad fina y } \\
\text { gruesa }\end{array}$ & $\begin{array}{c}\text { Ensamblado } \\
\text { con patrones } \\
\text { predeterminados }\end{array}$ & Dibujo técnico & Proceso de diseño \\
\hline $\begin{array}{l}\text { Construcciones- } \\
\text { Materiales }\end{array}$ & $\begin{array}{l}\text { Materias primas } \\
\text { Modelos } \\
\text { bidimensionales }\end{array}$ & $\begin{array}{c}\text { Modelos } \\
\text { tridimensionales } \\
\text { Medidas }\end{array}$ & Diseño CAD & $\begin{array}{c}\text { Fabricación de objetos } \\
\text { siguiendo el proceso } \\
\text { de diseño }\end{array}$ \\
\hline $\begin{array}{l}\text { Robótica y } \\
\text { sociedad }\end{array}$ & $\begin{array}{c}\text { Robots en su diario } \\
\text { vivir }\end{array}$ & $\begin{array}{c}\text { Tipos, papel y } \\
\text { clasificación de los } \\
\text { robots }\end{array}$ & $\begin{array}{c}\text { Uso de robots } \\
\text { Leyes de la robótica }\end{array}$ & $\begin{array}{c}\text { Robots y ética } \\
\text { Inteligencia artificial }\end{array}$ \\
\hline
\end{tabular}

Fuente: elaboración propia. 
de tecnología e informática, que es obligatoria en el sistema escolar, sino en otras áreas, pero que se pone a consideración, para que cada institución, docente y estudiante dé prioridad, según sus necesidades, a los temas aquí referidos, siempre y cuando su implementación contribuya a la mejora de la educación tecnológica.

\section{Conclusiones y trabajos futuros}

Este estudio presenta los resultados de un proceso de investigación documental centrada tanto en literatura como en información de páginas web actuales, donde se resaltan los hallazgos relevantes en robótica educativa, a partir de la caracterización de cinco enfoques y diez temáticas habilidades que se relacionan a partir de tres dimensiones y perspectivas del conocimiento. A su vez, las dimensiones y perspectivas hacen parte del currículo, ya que estas abordan de manera efectiva los estándares de aprendizaje requeridos en Colombia en el área de tecnología y en el proyecto "Currículos exploratorios en programación y uso de TIC", dado que cubre algunos temas ordenados en la Guía 30 y en los Estándares Básicos de Competencias en Lenguaje, Matemáticas, Ciencias y Ciudadanas (MEN, 2006; 2008). Adicionalmente, posibilita, con los debidos ajustes, replicarla a nivel internacional, según necesidades y requerimientos, ya que los objetivos de aprendizaje de este currículo abarcan el desarrollo de habilidades y conocimientos acordes a las condiciones actuales que precisan los estudiantes para su vida.

La educación en robótica en Colombia se ha ido incrementando y concretando en los últimos años, dado que el país, a nivel gubernamental y con empresas de índole privado, ha identificado la necesidad de formar estudiantes con un pensamiento más analítico, lógico y racional, capaces de estructurar y tomar decisiones respecto a situaciones problema o necesidades de manera sistemática, hasta encontrar una solución funcional y efectiva. Entonces, la robótica debe tener un espacio dentro del sis- tema educativo, dado que posibilita la motivación y el autoaprendizaje del estudiante.

Cabe aclarar que, para el caso de Colombia, actualmente se presenta un mayor enfoque de la robótica hacia el "uso del tiempo libre", ya que los cursos en robótica se dan en jornada complementaria o por centros de interés, con lo que se genera que las temáticas y el impacto se den a partir de proyectos que son entregables y mostrables en competencias, lo cual, si bien no resulta malo, no le da un sustento fuerte al área de tecnología, como tampoco posibilita un desarrollo pleno de la robótica, ni que esta se integre satisfactoriamente a las competencias que el Ministerio y la sociedad persiguen.

Este documento identifica y presenta cinco enfoques, según la intencionalidad del modelo de trabajo realizado por los diferentes estamentos internacionales y nacionales (Ministerios o Secretarías de Educación) y apoyados por diversos autores, a partir de casos de estudio, para abordar la robótica educativa. Esto sirvió de base para la creación de una propuesta curricular acorde a las necesidades colombianas donde se homogenizan ciertas temáticas y se legitima la robótica en la educación básica y media. Sin embargo, como no se tuvo en cuenta la efectividad e impacto de la propuesta en los estudiantes, en trabajos futuros sería pertinente indagar y analizar su incidencia en el aprendizaje de robótica.

Se puede identificar la preocupación de los diferentes gobiernos y entidades por incentivar la programación y el pensamiento computacional en la educación básica y media (Figura 1), ya que estas se consideran habilidades para el siglo XXI -fuente de futuros trabajos-. Por tal razón, se propone su integración con otras disciplinas y relaciones a partir de la robótica, y así darle flexibilidad en el proceso de aprendizaje, siendo que el modelo curricular propuesto se postula como un proceso abierto en su implementación, con la constante participación tanto del educando como del educador en roles activos. 
Finalmente, se presenta una nueva perspectiva de integración curricular enriquecida entre TI Robótica, sustentada en futuras experiencias sistematizadas en los diversos grados de escolaridad, ya que en Colombia no hay un estudio riguroso en este terreno, por lo cual resultaría interesante su ejecución. Además, esta propuesta proporciona una guía para la implementación de la robótica en las instituciones educativas útil para educadores, profesionales e in- vestigadores en el área de las tecnologías de la información y las comunicaciones, en conjunto con la educación, puesto que varios estudios han demostrado que la robótica tiene gran potencial como herramienta de enseñanza-aprendizaje interdisciplinar. Por lo anterior, el valor de la propuesta radica asimismo en su capacidad de ayudar a diseñar mejores planes de estudio y actividades que conlleven una mayor relación con el mundo por parte de los estudiantes.

\section{Referencias}

Alimisis, D., Moro, M. y Menegatti, E. (eds.) (2017). Educational robotics in the makers era. Springer. 10.1007/9783-319-55553-9

Alves da Costa, S., Pfeuti, M. de L. M. y Pereira de Castro C. N., S. (2014). As estratégias de ensino-aprendizagem utilizadas pelos docentes e sua relação com o envolvimento dos alunos. Revista Evidenciação Contábil \& Finanças, 2(1), 59-74. https://doi.org/10.18405/recfin20140104

Barrientos, A., Peñín, L. F., Balaguer, C. y Aracil, R. (1997). Fundamentos de robótica. McGraw-Hill.

Benitti, F. B. V. (2012). Exploring the educational potential of robotics in schools: A systematic review. Computers \& Education, 58(3), 978-988. https://doi.org/10.1016/j.compedu.2011.10.006

Bravo Sánchez, F. Á. y Forero Guzmán, A. (2012). La robótica como un recurso para facilitar el aprendizaje y desarrollo de competencias generales. Teoría de la Educación. Educación y Cultura en la Sociedad de la Información, 13(2), 120-136. http://www.redalyc.org/articulo.oa?id=201024390007

Bruner, J. S., González, J. M. I. y Palacios, J. (1988). Desarrollo cognitivo y educación. Morata.

Cantú, E. y Farines, J. M. (2007). Applying educational models in technological education. Education and Information Technologies, 12(3), 111-122. https://doi.org/10.1007/s10639-007-9038-4

Castiblanco, M. A., Sanabria Forigua, C. P. y Lora, M. A. (2015). Robótica educativa: un entorno tecnológico de aprendizaje que contribuye al desarrollo de habilidades. Pontificia Universidad Javeriana. https://repository.javeriana.edu.co/bitstream/handle/10554/17119/AcostaCastiblancoMarisol2015.pdf?sequence=1

Correa Ortiz, L. C., Vallejo Jiménez, M. M., Martínez Puerta, J. J. y Trujillo Posada, J. A. (2019). Herramienta de robótica educativa basada en Lego Mindstorms y VEX Robotics mediante software 3D y diseño mecatrónico. Risti. Revista Ibérica de Sistemas y Tecnologías de Información, 34(1), 1-19. https://doi.org/10.17013/risti.34.1-19

Fundación Compartir (2015). ¿Cómo enseñan los maestros colombianos en el área de Tecnología e Informática? 
García, M. A., Deco, C. y Collazos, C. A. (2015). Estrategias basadas en robótica para apoyar el pensamiento computacional. En XXII Congreso Argentino de Ciencias de la Computación (CACIC 2016) (pp. 1241-1250). RedUNCI. http://sedici.unlp.edu.ar/handle/10915/56279

Gómez-Álvarez, M. C., González Palacio, L., Manrique-Losada, B., Villada, B. y Arbeláez, S. (2019). Experiencias exitosas de enseñanza de programación y robótica en educación básica y media. En $14^{\text {th }}$ Iberian Conference on Information Systems and Technologies (CISTI) (pp. 1-6). IEEE. https://doi.org/10.23919/CISTI.2019.8760991

Gutiérrez Castro, B. (2016). La robótica educativa y su influencia en el aprendizaje colaborativo. Universidad Norbert Wiener.

Heiner, C. (2018). A robotics experience for all the students in an elementary school. En Proceedings of the $49^{\text {th }}$ ACM Technical Symposium on Computer Science Education - SIGCSE '18 (pp. 729-734). ACM Press. https://doi. org/10.1145/3159450.3159567

Hernández Sampieri, R., Fernández Collado, C. y Baptista Lucio, M. del P. (2010). Metodología de la investigación. McGraw-Hil.

Hrbacek, J., Kucera, M. y Strach, J. (2013). Teaching robot programming can be a new opportunity for technical subjects of study. En IEEE $11^{\text {th }}$ International Conference on Emerging eLearning Technologies and Applications (ICETA) (pp. 133-137). IEEE. https://doi.org/10.1109/ICETA.2013.6674418

Hurtado de Barrera, J. (2000). Metodología de la investigacion holística. Sypal.

Kandlhofer, M., Steinbauer, G., Hirschmugl-Gaisch, S. y Eck, J. (2014). A cross-generational robotics project day: Pre-school children, pupils and grandparents learn together. Journal of Automation, Mobile Robotics \& Intelligent Systems, 8(1), 12-19. https://doi.org/10.14313/JAMRIS_1-2014/2

Karp, T. y Maloney, P. (2013). Exciting young students in grades K-8 about STEM through an afterschool robotics challenge. American Journal of Engineering Education, 4(1), 39-54. http://eric.ed.gov/?q=robotic+\&ff1=dtyS ince_2006\&ff2=subRobotics\&pg=7\&id=EJ1057112

Khine, M. S., ed. (2017). Robotics in STEM Education. Redesigning the learning experience. Springer. https://doi. org/10.1007/978-3-319-57786-9

Lepuschitz, W., Merdan, M., Koppensteiner, G., Balogh, R. y Obdržálek, D. eds. (2018). Robotics in education. Latest results and developments. Springer. https://doi.org/10.1007/978-3-319-62875-2

MEN - Ministerio de Educación Nacional de Colombia (2006). Estándares básicos de competencias en lenguaje, matemáticas, cienciasy ciudadanas. https://www.mineducacion.gov.co/1621/articles-340021_recurso_1.pdf

MEN - Ministerio de Educación Nacional de Colombia (2008). Guía 30: “Orientaciones generales para la educación en tecnología. Ser competente en tecnología: juna necesidad para el desarrollo!". https://doi.org/978958-691-296-9 
MEN - Ministerio de Educación Nacional de Colombia (2013). Competencias TIC para el desarrollo profesional docente. Oficina de Innovación Educativa con Uso de Nuevas Tecnología. https://www.mineducacion.gov. co/1759/articles-339097_archivo_pdf_competencias_tic.pdf

Merdan, M., Lepuschitz, W., Koppensteiner, G. y Balogh, R. (eds.) (2017). Robotics in education. Research and practices for robotics in STEM education. Advances in Intelligent Systems and Computing. Springer. 10.1007/9783-319-42975-5

Merdan, M., Lepuschitz, W., Koppensteiner, G., Balogh, R. y Obdržálek, D. (eds.) (2020). Robotics in Education. Current Research and Innovations. Advances in Intelligent Systems and Computing. Springer. 10.1007/978-3030-26945-6

Mestres Naval, F.y Vives-Rego, J. (2011). Precisiones interdisciplinares y conceptuales de los términos cyborg, clon humano y robot. Ludus Vitalis, XIX(35), 235-238. http://www.ludusvitalis.org/textos/35/35_mestres_vivesrego.pdf

Meza Jaque, J. (2018). Modelo SAEP Seguimiento de los Aprendizajes desde Educación Parvularia. Academia del Hispanismo.

Monsalves, S. (2011). Estudio sobre la utilidad de la robótica educativa desde la perspectiva del docente. Revista de Pedagogia, 32, 81-117. http://saber.ucv.ve/ojs/index.php/rev_ped/article/view/1397

Montironi, M. A., Eliahu, D. S. y Cheng, H. H. (2015). A robotics-based 3D modeling curriculum for K-12 education. ASEE Annual Conference \& Exposition. https://doi.org/10.1826o/p.23443

Mubin, O., Stevens, C. J., Shahid, S., Mahmud, A. A. y Dong, J.-J. (2013). A review of the applicability of robots in education. Technology for Education and Learning, 1(1). https://doi.org/10.2316/Journal.209.2013.1.209-0015

Papert, S. (1980). Mindstorms children, computers, and powerful ideas. B. Books.

Pina-Calafi, A. (2017). Robótica educativa en educación primaria: ¿por qué y cómo? En S. Pérez A., G. Castellano P. y A. Pina C. (eds.), Propuestas de innovación educativa en la sociedad de la información (pp. 15-27). Adaya.

Pinto, M., Barrera, N. y Pérez, W. (2010). Uso de la robótica educativa como herramienta en los procesos de enseñanza. ${ }^{2}+D, 10(1), 15-23$. http://revistas.uptc.edu.co/revistas/index.php/ingenieria_sogamoso/article/viewFile/912/912

Pinto Santos, A., Díaz Carreño, J. y Camargo, C. (2016). Modelo espiral de competencias docentes tictActep aplicado al desarrollo de competencias digitales. Hekademos: Revista Educativa Digital, 19, 39-48. https://dialnet. unirioja.es/servlet/articulo?codigo $=6280715$

Quecedo Lecanda, R. y Castaño Garrido, C. (2002). Introducción a la metodología de investigación cualitativa. Revista de Psicodidáctica, 14, 5-39. http://www.redalyc.org/resumen.oa?id=17501402

Saha, S. K. (2010). Introducción a la robótica. McGraw-Hill. 
Scaradozzi, D., Sorbi, L., Pedale, A., Valzano, M. y Vergine, C. (2015). Teaching robotics at the primary school: An innovative approach. Procedia. Social and Behavioral Sciences, 174, 3838-3846. https://doi.org/10.1016/j.sbspro.2015.01.1122

Serrano Castaño, C. E. (2005). Modelo para la investigación documental. En Modelo integral para el profesional en ingeniería (cap. 2). Universidad del Cauca.

Štuikys, V. (2015). Smart learning objects for smart education in computer science. Springer. https://doi. org/10.1007/978-3-319-16913-2

Torres, M. L. (2015). Robótica como recurso didáctico. En II Congreso de Creatividad, Diseño y Comunicacion para Profesores y Autoridades de Nivel Medio. "Interfaces Palermo" 2014 (vol. 25, pp. 261-264). Universidad de Palermo. https://fido.palermo.edu/servicios_dyc/publicacionesdc/vista/detalle_articulo.php?id_libro=544\&id_ articulo $=11472$

Ucgul, M. y Cagiltay, K. (2014). Design and development issues for educational robotics training camps. International Journal of Technology and Design Education, 24(2), 203-222. https://doi.org/10.1007/s10798-013-9253-9

Viegas-D’Abreu, J. V. y Villalba-Condori, K. O. (2017). Education and Educative robotics. Revista de Educación a Distancia, 54(54), 2-13. https://doi.org/10.6018/red/54/11

Villalonga Gómez, C., Marta-Lazo, D. C.y Es, C. (2015). Modelo de integración educomunicativa de "apps" móviles para la enseñanza y aprendizaje. Pixel-Bit. Revista de Medios y Educación (enero), 1133-8482. 10.12795/pixelbit.2015.146.09 